\title{
Discounting pain in the absence of medical evidence is explained by negative evaluation of the patient
}

Lies De Ruddere ${ }^{1}$, Liesbet Goubert ${ }^{1}$, Michaël Stevens ${ }^{2}$, Amanda C de C Williams ${ }^{3}$, Geert Crombez ${ }^{1}$

${ }^{1}$ Department of Experimental-Clinical and Health Psychology, Ghent University, Ghent, Belgium

${ }^{2}$ Department of Experimental Psychology, Ghent University, Ghent, Belgium

${ }^{3}$ Research Dept of Clinical, Educational \& Health Psychology, University College London, London, United Kingdom

Corresponding author: Lies De Ruddere, Department of Experimental-Clinical and Health Psychology, Ghent University, Henri Dunantlaan 2, B-9000 Gent, Belgium. Tel: +32 (0)9 264 86 11, Fax: +32 (0)9 26464 89. Electronic mail may be sent to Lies.DeRuddere@ Ugent.be.

Text pages: 25

Figures: 2

Tables: 2

Key words: pain estimation, observers, pain behavior, contextual information 


\begin{abstract}
This study investigated the effect on observer responses of the presence/absence of information about medical evidence for pain and psychosocial influences on the patient's pain experience. Additionally, the moderating role of the patient's pain expressions and the mediating role of the observer's belief in deception and evaluation of the patient was examined. Sixty-two participants were presented videos of 4 patients, each accompanied by a vignette describing presence or absence of both medical evidence for the pain and psychosocial influences on the patient's pain. Participants estimated patients' pain, rated their own sympathy and inclination to help; they re-estimated patients' pain when the patient's selfreport of pain was provided. Finally, participants evaluated each patient as positive or negative, and the likelihood the patient was feigning pain. Participants gave lower ratings on pain, sympathy and help when medical evidence was absent. Further, in the presence of psychosocial influences, participants took patients' self-reported pain less into account. Next, only for patients expressing high intensity pain, information about both medical evidence and psychosocial influences were taken into account. Finally, the observer's evaluation of the patient and his/her belief in deception fully, respectively partially, explained the effect of medical evidence. The results indicate that discounting pain in the absence of medical evidence may involve negative evaluation of the patient. Further, the patient's pain expression is a moderating variable, and psychosocial influences negatively impact the degree to which patients' self-reports are taken into account. The results indicate that contextual information impacts on observer responses to pain.
\end{abstract}

Note: This is an uncorrected version of an author's manuscript accepted for publication. Copyediting, typesetting, and review of the resulting proofs will be undertaken on this manuscript before final publication. During production and pre-press, errors may be discovered that could affect the content. 


\section{Introduction}

Pain experiences are inherent to human life. Nevertheless, pain management often remains unsatisfactory $[3,36]$, especially when pain is chronic and/or when diagnosable pathology is absent $[3,37]$. When a clear medical explanation for pain is lacking, people in pain may feel frustrated and may feel that they are disbelieved by others $[1,21,30,42,45]$. Furthermore, those who observe people in pain may feel unable to provide adequate care when clear medical evidence for the pain is lacking [29].

Further insight into the social context in which an individual experiences pain for which there is no clear medical explanation is fundamental. Research demonstrated that observers attribute less pain to a patient $[5,6,12,38,39,41]$, feel less sympathy for the patient and are less inclined to help the patient [12] when clear medical evidence for the pain is lacking. Others' reactions towards the person with pain, such as pain estimation, feelings of sympathy and the inclination to help are important, as these responses may underlie pain management decisions and affect the wellbeing of the individual in pain [19].

At present, it is not known which mechanisms account for the effects of lacking medical evidence on observer responses. The absence of diagnosable pathology determining the patient's pain is considered as a risk factor for observers to impute to the person with pain the intention of feigning pain $[7,8,14]$. Additionally, there is preliminary evidence for the role of negative evaluation of the patient in the process of estimating a patient's pain where there is no clear medical evidence [41]. De Ruddere and colleagues [10,11] found that observer negative evaluations of patients appear to generate lower observer pain estimates.

Using a vignette paradigm with videos of low back pain patients performing four back straining activities, the present study had three objectives. First, we investigated the effects of absence versus presence of medical evidence and of psychosocial influences on observer responses (pain estimates, sympathy, and inclination to help). Although psychosocial factors 
and influences are common in pain suffering [4,16,28,31], it remains unclear whether their presence also affects observer responses. We also investigated whether information about both factors influences the degree to which observers take the verbal pain report of the patient into account when estimating the patient's pain. Second, we examined the patient's pain expressions as a moderating factor in the relationship between absence versus presence of medical evidence and psychosocial influences upon observer responses. We hypothesized that the effects of absence/presence of medical evidence and of psychosocial influences would be most pronounced when patients expressed high intensity pain. Following Tait and colleagues [40], observers might be more likely to take into account contextual information when judging high intensity pain. Third, we examined potential mechanisms underlying the effect of medical evidence on the observer responses. In particular, we investigated the mediating role of the observer's belief in deception by the patient, as well as the mediating role of the observer's judgment of how positively or negatively the patient is evaluated.

\section{Method}

\subsection{Participants}

Participants were recruited by an advertisement in local newspapers. Sixty-two (30 men, 32 women) individuals volunteered. To be eligible, participants had to be 18 years or older and speak Dutch fluently. Further, participants who indicated that they knew one of the patients shown on the videos were excluded. The mean age of the sample was 33.74 years $(S D=13.08 ;$ range $=19-64$ years $)$. About half of the participants were married, in a relationship or cohabiting $(58.1 \%)$, and about half of the participants had education beyond the age of $18(53.2 \%)$. Most were employed (67.8\%) and a quarter of the participants $(25.8 \%)$ were university or college students. The unemployment rate was $6.4 \%$. About one third of the participants $(N=18)$ reported having had no pain in the prior six months. The reported mean pain intensity during the past six months of the remaining 44 participants ('In the past six 
months, on the average, how intense was your pain rated on a 0 to 10 scale where 0 is "no pain" and 10 is "pain as bad as could be"?) was $4.00(S D=2.01$; range $=1-8)$. All participants were Caucasian. The study was approved by the ethical committee of the Faculty of Psychology and Educational Sciences of Ghent University.

\subsection{Design}

The experiment consisted of four phases (see Figure 1). In the first, pain estimation phase, participants were shown pictures of four different patients each accompanied by a vignette. The information in the vignettes was manipulated in a 2 x 2 within-subjects design. Vignettes described the presence or absence of (1) medical evidence for the pain, and (2) psychosocial influences upon the pain experience. After each picture, a video of the patient performing a pain-inducing activity was shown. Subsequently, participants estimated the patient's pain, and rated their own sympathy and inclination to help the patient with daily activities. Second, during the pain estimation after feedback phase, videos of the patients were presented again with the pain ratings of the patients themselves. Participants again estimated the patient's pain. Third, during the patient evaluation rating phase, pictures of the patients were shown and participants rated to what extent they judged the patients to be positive or negative. Fourth, during the deception rating phase, pictures of the patients were shown again and participants rated to what extent they thought the patient was feigning his or her pain.

\subsection{Stimuli}

\subsubsection{Videos and pictures}

The videos and pictures were selected from the Ghent Pain Videos of Daily Activities (GPAVIDA), consisting of videos displaying 34 chronic back pain patients (19 women, 15 men; $M_{\text {age }}=52$ years (range: $23-74 ; S D_{\text {age }}=12$ years) who performed four back straining movements. All patients reported chronic low back pain and were in (outpatient) treatment for 
pain at the University Hospital in Ghent. The patients were asked to execute four movements: 1) lying down on a bed and standing up, 2) sitting down on a chair and standing up, 3) taking a box from the ground, putting it on a table and then lifting it and replacing it on the ground, and 4) picking up marbles from the ground. Each movement was videotaped and every patient started the movement in upright position with the face directed to the camera. The videos display patients' full body pain behaviors, i.e., facial pain expression and active pain behavior (e.g., guarding, holding or rubbing). Further, patients provided verbal pain ratings on a numerical scale (0: no pain at all - 10: pain as bad as could be) after the performance of each movement.

For the present study, video sequences of four patients were selected. These patients were selected based on specific criteria. In particular, to ensure generalizability across gender, we selected two female patients and two male patients. To investigate effects of pain expression, two patients displaying a low level of pain and two patients displaying a high level of pain were selected based upon face validity (the videos were also coded to confirm the distinction between low and high levels of pain expression, see below). Furthermore, we also ensured that patients' age across the genders and across the two levels of pain expression were similar (see Table 1). After the experiment was conducted, the videos were coded in order to have additional evidence for the distinction between low and high intensity pain expression. In particular, pain expressions of all 34 patients were coded by a reliable rater by means of an adjusted coding system ${ }^{1}$, based upon the pain behavior coding manual of Sullivan and colleagues (the Pain Can Paradigm; unpublished manual). To calculate inter-rater reliability, $20 \%$ of the pain expressions were coded by a second independent rater. Each movement was coded for the presence of one or more of the key facial pain expressions [9,33,35] [(absent

\footnotetext{
${ }^{1}$ This coding scheme is particularly suitable for the levels of pain expressed by the patients in this study; it is not as comprehensive as the pain behavior coding manual of Sullivan et al. (2007), as the set up did not allow to make a fine grained coding of the facial pain expressions of the patients.
} 
(0), slightly present (1) distinctly present (2)]. Next, the presence (1) or absence (0) of active pain behavior (e.g., guarding, holding or rubbing) was coded per second. Interrater reliability was calculated according to the formula given by Ekman and Friesen [15] that assesses the proportion of agreement on actions recorded by two coders relative to the total number of actions coded as occurring by each coder.

Videos were presented by the INQUISIT Millisecond software package [22] on a 745 Dell Optiplex computer with a 75 HZ, 19-inch colour CRT monitor. The overall mean length of the video fragments presented in the study was $16 \mathrm{~s}(S D=7.36)$.

\subsubsection{Vignettes}

Vignettes described (1) the presence or absence of medical evidence for the pain and (2) the presence or absence of psychosocial influences upon the pain experience. Medical evidence in the vignettes was referred to as "a compressed nerve" or " inflammation of the ligaments". These operationalisations of medical evidence were discussed with a general practitioner in order to ensure that they are representative of attributions for low back pain. Vignettes describing the presence of psychosocial influences included "job stress and feelings of anxiety" or "relational problems and depressed mood". Feelings of anxiety, depression and emotional distress have been found to impact patients' pain experience [16].

These biomedical explanations and psychosocial influences were counterbalanced across patients and across vignettes. Information about 'medical evidence' and 'psychosocial influences' provided within the vignettes was embedded within broader information about the patient's name, age, job and number of children. This information was also counterbalanced across vignettes as well as across patients (see appendix A for examples of vignettes).

\subsection{Measures}

2.4.1. Pain scale, sympathy scale and inclination to help scale 
A numerical rating scale (0-10) was used by observers to estimate the patient's pain, their own inclination to help the patient with daily activities, and their sympathy for the patient. Zero indicated 'no pain at all', 'totally unwilling', and 'no sympathy at all' respectively; 10 indicated 'pain as bad as could be', 'totally willing', and 'a lot of sympathy' respectively.

\subsubsection{Evaluation scale}

The extent to which the patient was judged to be positive or negative was assessed by a 21point scale ('Please indicate your current evaluation of each person on the scale' $-10=$ very negative, $0=$ neutral, $10=$ very positive) .

\subsubsection{Deception scale}

The extent to which the participant thought the patient was feigning her or his pain was measured by an 11-point numerical rating scale ( 0 indicated 'not at all', 10 indicated 'a lot').

\subsection{Procedure}

\subsubsection{Rating phase}

In the experiment room, the participant was seated in front of a computer at a distance of about $60 \mathrm{~cm}$ from the screen. Participants were informed that this study examined people's impression formation of others in pain. Participants were told that (1) written information about four people and their pain complaints would be given, followed by 2) presentation of video fragments of these people on the computer screen. Written informed consent was obtained. When the participant pressed ENTER on the PC keyboard, a (neutral) picture of a patient was shown combined with one vignette. When the participant pressed ENTER again, the video fragment of the same patient performing a pain-inducing activity was presented. This procedure was repeated with the video fragments of the three other patients. Vignettes were counterbalanced across the four patients. Within each participant, the four patients were presented with each of the vignettes describing 1) medical evidence and psychosocial influences, 2) no medical evidence and psychosocial influences, 3) medical evidence and no 
psychosocial influences or 4) no medical evidence and no psychosocial influences. Each patient was shown four times (i.e., each movement was shown once). In sum, 16 videos were shown in random order to the participants. After the presentation of each video, a blank screen appeared and participants were requested to estimate the patient's pain, and to rate their sympathy for the patient and their inclination to help the patient.

\subsubsection{Pain estimation after feedback phase}

Participants were presented one video of each patient (four videos in total). For each participant, all four patients were performing the same movement in this phase. The movement that all four patients were performing was counterbalanced across participants. Participants were provided with self-reported pain intensity ratings of the patients and were once again asked to estimate patients' pain.

\subsubsection{Patient evaluation rating phase}

One (neutral) picture of each patient was shown to the participant who rated the extent to which she/he judged the patient to be negative or positive.

\subsubsection{Deception rating phase.}

One (neutral) picture of each patient was shown to the participant who rated the extent to which she/he thought the patient was feigning pain. At the end of the experiment, the participant was requested to fill out a second informed consent after revealing the true purpose of the study.

\subsection{Statistical analyses}

Outcome variables were participants' estimates of pain ( 'pain'), sympathy ( 'sympathy') and inclination to help ( 'help') as well as the absolute difference between the pain rating of the patient and the pain estimation of the observer after the patient's own pain rating was provided during the 'pain estimation after feedback phase' ('discrepancy'). As each patient was presented four times and the ratings (pain, sympathy, help) for each presentation were 
highly correlated (pain: $r=.84$, sympathy: $r=.90$, inclination to help: $r=.91 ; p<.001$ ), a mean score for each outcome variable was calculated per patient. Next, the presence/absence of medical evidence ('medical evidence') and psychosocial influences ('psychosocial influences') as well as the level of pain expressed by the patient (high or low 'pain expression') were included in the analyses as independent variables. Further, the observer's evaluation of the patient ('evaluation') as well as the observer's belief in deception ('deception') were the potential mediating variables in the relationship between presence/absence of medical evidence and the observer responses (pain, sympathy and help).

The factors in the present study were manipulated partially within and partially between subjects. Within subjects, each level of 'medical evidence' and 'psychosocial factors' was combined with only one of the two levels of 'pain expression'. Between subjects, each level of 'medical evidence' and 'psychosocial factors' was combined with each level of 'pain expression'. Because this type of factorial design cannot be handled by classical repeated measures analyses, the results were analyzed using linear mixed effects models as implemented in the $\mathrm{R}$ package nlme [32]. Linear mixed effects models account for the correlations in within-subjects data by estimating subject-specific deviations (or random effects) from each population-level factor (or fixed factor) of interest (see [46] for an elaboration).

Each analysis consisted of three steps. First, all relevant factors and interactions were entered in the model as fixed factors. In the second step, we assessed whether it was necessary to add a random effect for each of the fixed factors in the analysis: if a random effect significantly increased the fit of the model, it was included in the final model. In the third step, we inspected the ANOVA table of the final model and tested specific hypotheses about possible main effects or interactions (see $[10,43]$ for a similar approach). When testing 
specific hypotheses, standardized regression weights were reported as a measure of effect size.

Mediation analyses followed the procedure outlined by Judd and colleagues [24]. First, we investigated (by means of a paired sample t-test) whether the scores on the potential mediating variables were lower (evaluation) and higher (deception) when medical evidence was absent compared to when medical evidence was present. Second, we computed difference scores for the potential mediating variables (for evaluation: the difference between 1) evaluation when medical evidence is present and 2) evaluation when medical evidence is absent; for deception: the difference between 1) deception when medical evidence is absent and 2) deception when medical evidence is present). Next, we examined (by means of regression analyses) whether these difference scores were related to the differences (between presence and absence of medical evidence) in the outcome variables, pain, help, sympathy and discrepancy.

3. Results

\subsection{Results concerning the coding of the videos}

Acceptable inter-rater reliability was achieved for facial pain expression (.66) and active pain behavior (.89). The scores on facial pain expression could range from $0-2$ and the scores on active pain behavior were calculated by summing the seconds in which the patient was showing active pain behavior. The scores on facial pain expression and active pain behavior are presented in Table 1. Furthermore, we provided information on quartiles to indicate how the selected patients related to the larger patient sample $(N=34)$ regarding pain expression scores (see Table 1$)^{2}$.

\section{- INSERT TABLE 1 ABOUT HERE -}

\footnotetext{
${ }^{2}$ For more information on the Ghent Pain Videos of Daily Activities (G-PAVIDA) (also regarding the use of the videos for research purposes) please contact Lies De Ruddere (Lies.DeRuddere@UGent.be) or Liesbet Goubert (Liesbet.Goubert@UGent.be).
} 
3.2. Impact of presencelabsence of medical evidence and of psychosocial influences on the observer responses pain, help and sympathy

For the analyses of the outcomes pain, help, and sympathy, random effects of medical evidence, psychosocial influences, and pain expression were included. The results indicated a significant main effect of pain expression on pain estimates $(F(1,923)=206.59, p<.001, \beta=$ $0.92)$, on help $(F(1,923)=87.44, p<.00, \beta=0.63)$, and on sympathy $(F(1,923)=33.45, p<$ $.001, \beta=0.45)$ : participants reported higher pain ratings, more sympathy and more inclination to help when the patient expressed high intensity pain. Further, the results revealed a significant main effect of medical evidence on pain $(F(1,923)=25.40, p<.001, \beta=0.30)$, help $(F(1,923)=23.14, p<.001, \beta=0.31)$, and sympathy $(F(1,923)=22.56, p<.001, \beta=$ 0.32), such that participants reported lower pain ratings, less sympathy, and less inclination to help when medical evidence for pain was absent. No significant effect of psychosocial influences on pain $(F(1,923)=0.81, p=.376, \beta=-0.05)$, help $(F(1,923)=0.75, p=.387, \beta=$ -0.04), or sympathy $(F(1,923)=0.01, p=.940, \beta=0.004)$ was found. Further, the medical evidence $\mathrm{x}$ psychosocial influences interaction was not significant for pain $(F(1,923)=0.11, p$ $=.741)$, sympathy $(F(1,923)=2.83, p=.093)$, or help $(F(1,923)=1.65, p=.199)$.

3.3. Impact of presence/absence of medical evidence and of psychosocial influences on the degree to which the observer takes the patient's self report of pain into account

For the analyses of discrepancy between the patient's pain rating and the participant's pain estimate after being informed about the patient's rating, only a random intercept was necessary. There was a main effect of pain expression $(F(1,176)=29.78, p<.001, \beta=-0.53)$, indicating larger discrepancies when pain expression was low. There was a marginally significant effect of medical evidence $(F(1,176)=3.34, p=.069, \beta=-0.18)$, indicating a trend towards larger discrepancies when medical evidence was absent. There was an effect of psychosocial influences $(F(1,176)=9.35, p=0.003, \beta=0.30)$, indicating larger (absolute) 
discrepancy between the pain ratings of the patients and the estimates by observers when psychosocial influences were present. None of the two-way interactions was significant, neither was the three-way interaction. The means and standard errors of participants' scores on discrepancy for each cell are provided in Table 2.

3.4. The moderating role of the patient's pain expression in the relationship between medical evidence and psychosocial influences, and observer responses pain, help and sympathy

Pain expression did not moderate the effect of medical evidence on pain $(F(1,923)=1.23$, $p=.268)$, sympathy $(F(1,923)=0.88, p=.349)$, or help $(F(1,923)=2.54, p=.111)$. Further, there was no pain expression $\mathrm{x}$ psychosocial influences interaction on pain $(F(1,923)=0.03, p$ $=.858)$, sympathy $(F(1,923)=0.42, p=.516)$ or help $(F(1,923)=1.32, p=.252)$. However, the three-way interaction effect between pain expression, medical evidence and psychosocial influences was significant for pain $(F(1,923)=3.95, p=.047)$ and for help $(F(1,923)=4.38$, $p=.036)$ but not for sympathy $(F(1,923)=2.44, p=.118)$. This three-way interaction effect for pain and help indicated that the influence of medical evidence and psychosocial influences was dependent upon the level of pain expressed by the patient. The interaction did not invalidate the main effects of pain expression or medical influence: within each cell the main effects of pain expression and medical influence were present and in the same direction.

For estimates of pain, the medical evidence x psychosocial influences interaction was not significant for patients expressing low intensity pain $(F(1,923)=2.11, p=.145)$; but it was marginally significant for patients expressing high intensity pain $F(1,923)=3.36, p=.067$ ): when there were no psychosocial influences in case the patients were expressing high intensity pain, the effect of medical evidence was more pronounced $(\mathrm{F}(1,923)=22.78, p<$ $0.001, \beta=0.48)$ than when the psychosocial influences were present $(\mathrm{F}(1,923)=4.36, p=$ $0.037, \beta=0.22)$ 
Further, the analyses revealed that for help, the medical evidence $\mathrm{x}$ psychosocial influences interaction was not significant for patients expressing low intensity pain $(F(1,923)$ $=1.28, p=.26$ ); but it was significant for patients expressing high intensity pain $F(1,923)=$ $6.03, p=0.014)$ : when there were no psychosocial influences, the effect of medical evidence was more pronounced $(F(1.923)=30.26, p<0.001, \beta=0.55)$ than when the psychosocial influences were present $(F(1,923)=3.72, p=0.054, \beta=0.21)$ (see Figure 1). The means and standard errors of the participants' scores on pain, sympathy and help for each cell are provided in Table 2 .

\section{- INSERT FIGURE 1 ABOUT HERE - \\ - INSERT TABLE 2 ABOUT HERE -}

3.5. Mediating role of the participant's evaluation of the patient in the relationship between medical evidence and observer responses pain, help and sympathy

Analyses revealed a significant difference between patient evaluation when medical evidence was present and patient evaluation when medical evidence was absent $(t(61)=5.18$, $p<.001$ ), indicating more positive evaluation scores when medical evidence was present compared to when medical evidence was absent $\left(M_{\text {medical evidence }}=3.37 ; M_{\text {nomedicalevidence }}=\right.$ 0.97). Further, regression analyses revealed that the difference scores for evaluation (the difference between evaluation when medical evidence is present and evaluation when medical evidence is absent) were related to the difference scores for pain $(t(61)=4.11, p<.001 ; \beta=$ $0.490)$, help $(t(61)=6.32, p<.001 ; \beta=0.658)$, and sympathy $(t(61)=8.40, p<.001 ; \beta=$ 0.746). These results indicate that the effect of medical evidence upon pain, help and sympathy was mediated by the participant's evaluation of the patient. Furthermore, the residual effects of the presence/absence of medical evidence were not longer significant for pain $\left(\beta_{\text {intercept }}=.334, p=.126\right)$, help $\left(\beta_{\text {intercept }}=.264, p=.114\right)$ and sympathy $\left(\beta_{\text {intercept }}=.184\right.$, 
$p=.184)$ when controlling for the mediating role of patient evaluation, indicating full mediation by the participant's evaluation of the patient.

3.6. Mediating role of the participant's belief in deception in the relationship between medical evidence and observer responses pain, help and sympathy

Analyses revealed a significant difference between the participant's belief in deception when there was no medical evidence for the pain compared to when there was medical evidence for the pain $(t(61)=4.61, p<.001)$, indicating higher deception scores when medical evidence was absent $\left(M_{\text {medical evidence }}=2.75 ; M_{\text {nomedicalevidence }}=4.32\right)$. Further, regression analyses revealed that the difference scores for deception (the difference between deception when medical evidence is absent and deception when medical evidence is present) were related to the difference scores for pain $(t(61)=2.27, p<.05)$, help $(t(61)=3.78, p<$ $.001)$, and sympathy $(t(61)=4.64, p<.001)$. Although decreased, the residual effects of the presence/absence of medical evidence remained significant for pain $\left(\beta_{\text {intercept }}=0.575, p<\right.$ $.05)$, help $\left(\beta_{\text {intercept }}=0.503, p<.01\right)$ and sympathy $\left(\beta_{\text {intercept }}=0.436, p<.05\right)$ when controlled for the mediating role of deception, indicating only a partial mediation by the participant's belief in deception ${ }^{3}$.

\section{Discussion}

This study investigated the effects of information about medical evidence for pain and of psychosocial factors on observer responses and on the degree to which observers take the selfreported pain intensity of the patient into account. Further, we investigated whether the effects of medical evidence and psychosocial influences is moderated by the patient's level of pain expression. Further, the study examined whether the effect of the absence of medical evidence upon observer responses is explained by 1) the observer's negative evaluation of the patient

\footnotetext{
${ }^{3}$ The scores on evaluation were not significantly related to the scores on deception $(r=-.23, p=.075)$. Further, the scores on evaluation were not significantly related to the scores on pain $(r=.24, p=.063)$ and help $(r=.22$, $p=.089)$, but were positively related to the scores on sympathy $(r=.47, p<.001)$. Equally, the scores on deception were not related to the scores on pain $(r=-.19, p=.143)$ and help $(r=-.07, p=.588)$, but were negatively related to the scores on sympathy $(r=-.28, p<.001)$.
} 
and 2) the observer's belief in deception. Results indicated that participants ascribed lower pain, felt less sympathy for and were less inclined to help the person with pain when medical evidence for the pain was absent. Further, when estimating the patient's pain, participants who were told that the patient's pain experience was influenced by psychosocial factors took the patient's self report into account less. Next, results revealed that information about both medical evidence for pain and psychosocial influences upon the pain experience were taken into account in relation to the inclination to help the patient expressing high intensity pain. Further, the results indicated that the observer's negative evaluation of the patient fully mediated, and his/her belief in deception partially mediated, the relationship between the absence of medical evidence and the participant responses.

The design of this study allowed investigation of the influence of medical evidence on participant responses when actual patients showing full body pain behavior were observed. The results are in line with the findings of several vignette studies that presented written descriptions of fictitious patients to the participants [5,6,38,39,41]. Only recently, De Ruddere and colleagues [12] demonstrated the influence of medical evidence by means of videos displaying the facial pain expression of actual patients. In accordance with De Ruddere and colleagues [12], the current findings did not reveal an effect of psychosocial influences on observer responses. However, in the current study, information about psychosocial influences on the patient's pain influenced the degree to which the pain report of the patient was taken into account. One potential explanation may be that participants considered the patient's selfreported pain as under psychosocial influence: participants might have assumed that when psychosocial influences were present, patients were in some way biased in their pain ratings.

Further, our study was designed to examine the moderating role of one influential factor: the level of pain expressed by the patient $[17,47]$. Although the underlying mechanisms remain unclear, Tait and colleagues [40] indicate that contextual information has more impact 
on pain estimates when observers judge high intensity pain. For example, Tait and colleagues [40] describe the study of Chibnall and Tait [5], which showed that the effect of the contextual factor (presence/absence of medical evidence) on observer pain estimates was more pronounced for patients reporting high intensity pain. With regard to our findings, absence of medical evidence had a smaller impact upon the observer's inclination to help the patient when psychosocial factors influenced the patient's pain and when the patient was expressing high intensity pain. Although the results do not allow a full interpretation, they suggest that observers take contextual cues more into account when estimating high intensity pain. One potential explanation for this finding may be that pain expression was sufficiently high, given cultural norms tending towards stoicism, that observers' suspicions were aroused and they searched for contextual information to help interpretation [8,47].

Our study was also designed to identify putative underlying mechanisms accounting for the effect of medical evidence. Considerable research indicates that observer responses towards others in pain are influenced by the observer's evaluation of the patient $[5,10,11$, 38,39]. For example, De Ruddere and colleagues [11] showed that observers attribute less pain to patients when they evaluate these patients more negatively. The present study reveals that the absence of medical evidence negatively affects the evaluation of patients. Further, the results provided evidence that this effect explained the observer's tendency to discount pain for which there is no clear medical evidence. This finding extends the preliminary findings of Taylor and colleagues [41], who found that nurses evaluated patients more negatively when there was no medical evidence for their pain. In many clinical settings, chronic pain patients are considered as 'difficult' patients [44], that is, they are negatively evaluated. The findings may reflect a general dislike of the patient by participants, due to the inability of the participants to identify with people with pain for which there is no clear medical explanation. In particular, it may be that the participants evaluated the patients more negatively due to a 
lack of knowledge about pain in the absence of clear medical evidence. For example, models of acute pain might dominate thinking about pain, including chronic pain. Although many individuals suffer from pain in the absence of disease or damage [20,23,26,27,34], many individuals still believe that pain should always be proportional to disease or damage $[13,18]$.

In addition to the observer's evaluation of the patient, the study indicated a second potential underlying mechanism: the observer's belief that the patient feigned her or his pain. Following Craig and colleagues [7,8], absence of diagnosable pathology associated with the patient's pain is a risk factor for observers to impute to the patient an intention of feigning pain. Our findings indicate that this mechanism partially underlies the relationship between absence of clear medical evidence for the pain and the observer's lower pain estimates, less sympathy and less inclination to help. Nevertheless, given the partial mediation, the findings suggest that a belief in malingering or deception is not the only mechanism explaining the detrimental effects of the absence of medical evidence upon observer responses. Instead, the results indicate the observer's evaluation of the patient as the key mechanism.

The findings of the present study may have some clinical implications. First, the results suggest that people for whom no medical evidence for their pain is available are negatively evaluated by observers. The results further indicate that this effect may be the reason why observers attribute less pain, feel less sympathy and are less inclined to help with daily activities. All this may impact the wellbeing of people with pain. Lower pain estimates, less sympathy and less inclination to help may negatively affect the observer's behavior towards the individual with pain (e.g., help giving). Second, suspecting deception when a medical explanation for the pain is lacking may lead to stigmatizing individuals with pain for which there is no clear medical explanation. For example, stigmatization may occur to the extent of believing the individuals to be malingering. This, in turn, has important repercussions for the wellbeing and identity of the people with pain [21]. Third, the results suggest that the patient's 
self-reported pain is taken less into consideration by the observer when information is provided that the pain is influenced by psychosocial factors, such as marital dissatisfaction or job stress. The patient's self-report is, with nonverbal pain behavior, an important cue for others' estimation of their pain [25], and informs appropriate decisions on pain treatment. Fourth, the results suggest that observers take information about psychosocial influences into account predominantly for high intensity pain. This finding may suggest that the observation of patients expressing high intensity pain made observers more sensitive to the information about both medical evidence and psychosocial influences.

Some limitations, each of which points to directions for future research, deserve attention. First, the vignette methodology may lack ecological validity; observer reports of their reactions to a vignette and picture/video may not predict their behavior in real life. Ways of bridging this gap are challenging for the researcher, but crucial for understanding clinical situations. Second, additional actual behavioral measures of help might strengthen the ecological validity of the results, as self-report is prone to social desirability effects. Third, the results from our participant observers may differ significantly from the beliefs and behavior of professional health care providers in relation to pain, medical and psychosocial influence on pain, and evaluation of patients. Fourth, although video sequences of actual patients with chronic low back pain were used in the study, one may question whether the four patients are representative of the full population of patients with pain. Fifth, the psychosocial influences described in our vignettes may have been weak compared with the psychosocial issues (e.g., clinical mood or anxiety disorders) that are often prominent in pain patients [2]. Therefore, future research may benefit from examining the influence of the comorbidity of pain with clinical mental disorders on observer responses. Sixth, future research may benefit from examining whether the patient's pain expression impacts on the mediating role of the participant's evaluation of the patient and/or his or her belief in deception. 


\section{Acknowledgments}

This study was supported by a grant from the Fund for Scientific Research (FWO) - Flanders (G.0299.09). The authors would like to thank Veerle Dubuy for her help with data collection and input of the data. There are no conflicts of interest that may arise as a result of the research presented in this manuscript. 


\section{References}

[1] Allegretti A, Borkan J, Reis S, Griffiths F. Paired interviews of shared experiences around chronic low back pain: classic mismatch between patients and their doctors. Fam Pract 2010;27:676-683.

[2] Beesdo K, Jacobi F, Hoyer J, Low NCP, Höfler M, Wittchen H-U. Pain associated with specific anxiety and depression disorders in a nationally representative population sample. Soc Psychiat Epidemiol 2010;45:89-104.

[3] Breivik H. Opioids in chronic non-cancer pain, indications and controversies. Eur J Pain 2005;9:127-160.

[4] Carragee EJ. Persistent low back pain. N Engl J Med 2005;352:1891-1898.

[5] Chibnall JT, Tait RC. Observer perceptions of low back pain: effects of pain report and other contextual factors. J Appl Soc Psychol 1995;25:418-439.

[6] Chibnall JT, Tait RC. Social and medical influences on attributions and evaluations of chronic pain. Psychol Health 1999;14:719-729.

[7] Craig KD, Badali MA. Introduction to the special series on pain deception and malingering. Clin J Pain 2004;20:377-382.

[8] Craig KD, Hill ML, McMurtry BW. Detecting deception and malingering. In: Block AR, Kremer EF, Fernandez E, editors. Handbook of Pain syndromes. Biopsychosocial perspectives. New Jersey, London: Lawrence Erlbaum Associates, 1999.

[9] Craig KD, Prkachin KM, Grunau RE. The facial expression of pain. In: Turk DC, Melzack R, editors. Handbook of pain assessment, second edition. New York, NY: The Guilford Press, 2001. pp. 153-169. 
[10] De Ruddere L, Goubert L, Prkachin KM, Stevens MAL, Van Ryckeghem DML, Crombez G. When you dislike patients, pain is taken less seriously. Pain $2011 ; 152: 2342-2347$.

[11] De Ruddere L, Goubert L, Vervoort T, Kappesser J, Crombez G. The impact of being primed with social deception upon observer responses to others' pain. Pain in press.

[12] De Ruddere L, Goubert L, Vervoort T, Prkachin KM, Crombez G. We discount the pain of others when pain has no medical explanation. J Pain in press.

[13] Eccleston C, Crombez G. Worry and chronic pain: A misdirected problem solving model. Pain 2007;132:233-236.

[14] Eccleston C, Williams ACdeC, Rogers WS. Patients' and professionals' understandings of the causes of chronic pain: blame, responsibility and identity protection. Soc Sci Med 1997;45:699-709.

[15] Ekman P, Friesen W. Investigator's guide to the facial action coding system. Palo Alto, CA: Consulting Psychologists Press, 1978.

[16] Gatchel RJ, Peng YB, Peters ML, Fuchs PN, Turk DC. The biopsychosocial approach to chronic pain: scientific advances and future directions. Psychol Bull 2007;133:581624.

[17] Goubert L, Craig KD, Buysse A. Perceiving others in pain: experimental and clinical evidence on the role of empathy. In: Ickes W, Decety J, editors. The Social Neuroscience of Empathy. Cambridge, MA: MIT Press, 2009.

[18] Goubert L, Crombez G, De Bourdeaudhuij I . Low back pain, disability and back pain myths in a community sample: prevalence and interrelationships. Eur J Pain 2004;8:385-394. 
[19] Hadjistavropoulos T, Craig KD, Duck S, Cano A, Goubert L, Jackson PL, Mogil JS, Rainville P, Sullivan M, Williams ACdeC, Vervoort T, Fitzgerald, T.D. A biopsychosocial formulation of pain communication. Psychol Bull 2011; 910-939.

[20] Hiller W, Rief W, Brähler E. Somatization in the population: from mild bodily misperceptions to disabling symptoms. Soc Psychiatry Psychiatr Epidemiol 2006;41:704-712.

[21] Holloway I, Sofaer-Bennett B, Walker J. The stigmatization of people with chronic back pain. Disab Rehab 2007;29:1456-1464.

[22] Inquisit 3.0.4.0 computer software. Seattle, WA: Millisecond Software, 2009.

[23] Jacobi F, Wittchen HU, Hölting C, Höfler M, Pfister H, Müller N, Lieb R. Prevalence, co-morbidity and correlates of mental disorders in the general population: results from the German Health Interview and Examination Survey (GHS). Psychol Med 2004;34:597-611.

[24] Judd CM, Kenny DA, McClelland GH. Estimation and testing mediation and moderation in within-subject designs. Psychol Methods 2001;6:115-134.

[25] Kappesser J, Williams ACdeC, Prkachin KM. Testing two accounts of pain underestimation. Pain 2006;124:109-116.

[26] Lahmann C, Henningsen P, Noll-Hussong M. Somatoform pain disorder - overview. Psychiatr Danub 2010;22:453-458.

[27] Lieb R, Pfister H, Mastaler M, Wittchen HU. Somatoform syndromes and disorders in a representative population sample of adolescents and young adults: prevalence, comorbidity and impairments. Acta Psychiatr Scand 2000;101:194-208.

[28] Linton SJ: A review of psychological risk factors in back and neck pain. Spine 2000;25:1148-1156. 
[29] Matthias MS, Parpart AL, Nyland KA, Huffman MA, Stubbs DL, Sargent C, Bair MJ. The patient-provider relationship in chronic pain care: providers' perspective. Pain Med 2010;11:1688-1679.

[30] Peters S, Stanley I, Rose M, Salmon P. Patients with medically unexplained symptoms: sources of patients' authority and implications for demands on medical care. Soc Sci Med 1998;46:559-565.

[31] Pincus T, Burton AK, Vogel S, Fiels AP. A systematic review of psychological factors as predictors of chronicity/disability in prospective cohorts of low back pain. Spine 2002;27:E109-E120.

[32] Pinheiro JC, Bates DM. Mixed-effects models in S and S-PLUS. New York: Springer-Verlag, 2000.

[33] Prkachin KM. The consistency of facial expressions of pain: a comparison across modalities. Pain 1992;51:297-306.

[34] Rief W, Hessel A, Braehler E. Somatization symptoms and hypochondriacal features in the general population. Psychosom Med, 2001;63:595-602.

[35] Rocha E, Prkachin KM, Beaumont SL, Hardy C, Zumbo BD. Pain reactivity and illness behavior in kindergarten-aged children. J Pediatr Psychol 2003;28:47-57.

[36] Sinatra R. Causes and consequences of inadequate management of acute pain. Pain Med 2010;11:1859-1871.

[37] Spacek A. Modern concepts of acute and chronic pain management. Biomed Pharmacother 2006;60:329-335.

[38] Tait RC, Chibnall JT. Observer perceptions of chronic low back pain. J Appl Soc Psychol 1994;24:415-431.

[39] Tait RC, Chibnall JT. Physician judgments of chronic pain patients. Soc Sci Med $1997 ; 45: 1199-1205$. 
[40] Tait RC, Chibnall JT, Kalauokalani D. Provider judgments of patients in pain: seeking symptom certainty. Pain Med 2009;10:11-34.

[41] Taylor AG, Skelton JA, Butcher J. Duration of pain condition and physical pathology as determinants of nurses' assessments of patients in pain. Nurs Res 1984;33:4-8.

[42] Toye F, Barker K. 'Could I be imagining this?' - the dialectic struggles of people with persistent unexplained back pain. Disab Rehab 2010;32:1722-1732.

[43] Verbruggen F, Aron AR, Stevens MA, Chambers CD. Theta burst stimulation dissociates attention and action updating in human inferior frontal cortex. Proc Nat Acad Sci 2010;107:13966-13971.

[44] Wasan AD, Wootton J, Jamison RN. Dealing with difficult patients in your pain practice. Reg Anesth Pain Med 2005;30:184-192.

[45] Werner A, Malterud K. It is hard work behaving as a credible patient: encounters between women with chronic pain and their doctors. Soc Sci Med 2003;57:14091419.

[46] West BT, Welch KB, Galecki AT. Linear Mixed Models: A practical guide using statistical software. London: Chapman \& Hall/CRC, 2007.

[47] Williams ACdeC. Facial expression of pain: An evolutionary account. Behav Brain Sci 2002;25:439-488. 
Table 1. The age of each patient and for each patient: 1) the scores on facial pain expression averaged across the four video sequences, 2) the active pain behavior, averaged across the four video sequences, 3 ) the mean duration of the video sequences and 4) patients' self reported pain ratings for each video sequence.

\begin{tabular}{ccccccccc}
\hline patient & age & facial pain expression & active pain behavior & duration & pain 1 & pain 2 & pain 3 & pain 4 \\
\hline FL & 65 & $0.5(2)$ & $8.25(3)$ & 11.55 s (2) & 7 & 4 & 6 & 7 \\
ML & 55 & $0.5(2)$ & $7(2)$ & 13.55 s (2) & 7 & 7 & 6 & 7 \\
FH & 46 & $1(4)$ & $11.25(3)$ & 20.25 s (4) & 8 & 8 & 8.5 & 8.5 \\
MH & 63 & $1(4)$ & $16.75(4)$ & 18.71 s (3) & 7 & 5.5 & 7 & 7
\end{tabular}

Note 1. In the column 'patient', the first initials refer to the gender of the patients $(\mathrm{F}=$ female, $\mathrm{M}=\mathrm{male})$ and the second initials refer to the level of pain expression that is displayed by the patient (based on face validity; $\mathrm{L}=$ low pain expression; $\mathrm{H}=$ high pain expression).

Note 2. Pain 1, pain 2, pain 3 and pain 4 refer to patients' self reported pain ratings for each video sequence.

Note 3. The quartile with regard to the scores of the 34 patients of the G-PAVIDA each patient fitted in is provided between brackets.

Note 4. The scores on active pain behavior of patient FL and patient FH fitted within the same quartile (based on the larger sample; $N=34$ ). However, the mean score on active pain behavior for the two patients in the 'low pain expression' group $\left(M_{\text {low }}=7.63\right)$ fitted within the $2^{\text {nd }}$ quartile while the mean score on active pain behavior for the two patients in the 'high pain expression' group $\left(M_{\text {high }}=14\right)$ fitted within the $4^{\text {th }}$ quartile. 
Table 2. Means (and stardard errors) of the participants' scores on pain, sympathy, help and discrepancy for each cell.

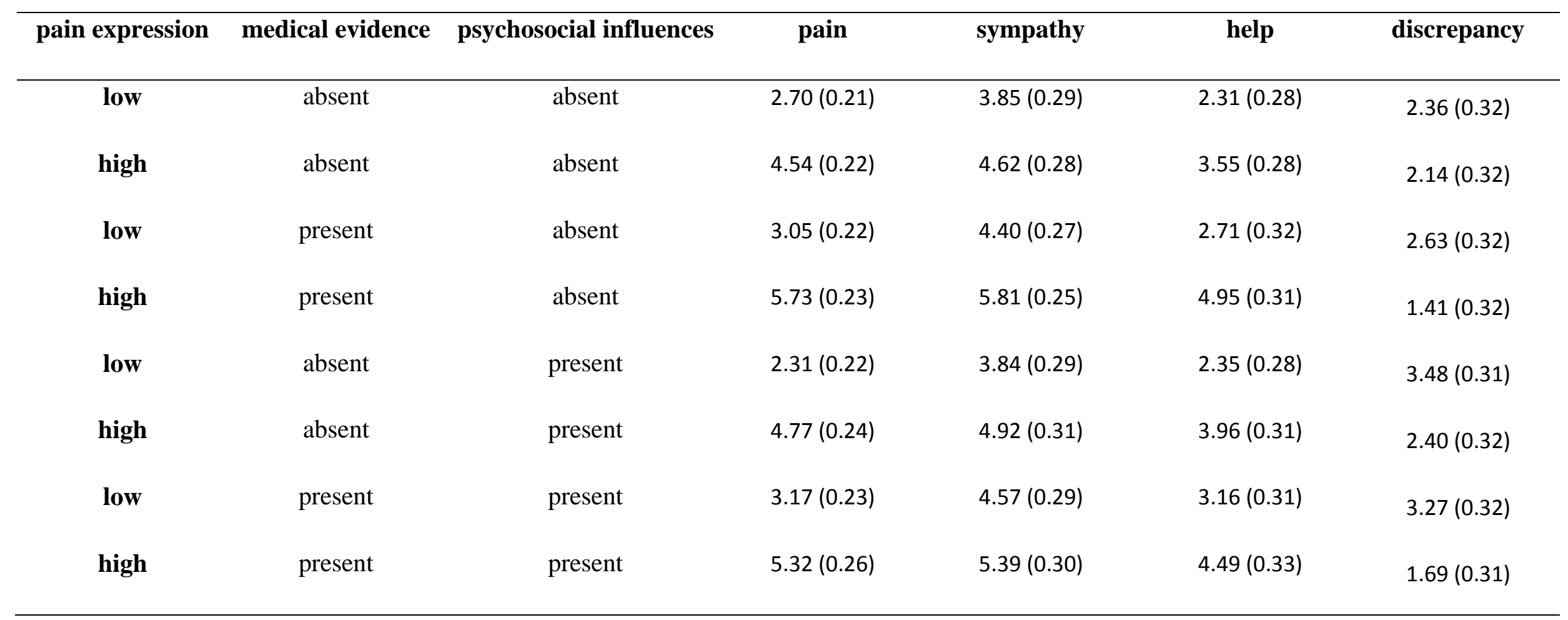


Figure 1. Flow diagram of the study design.

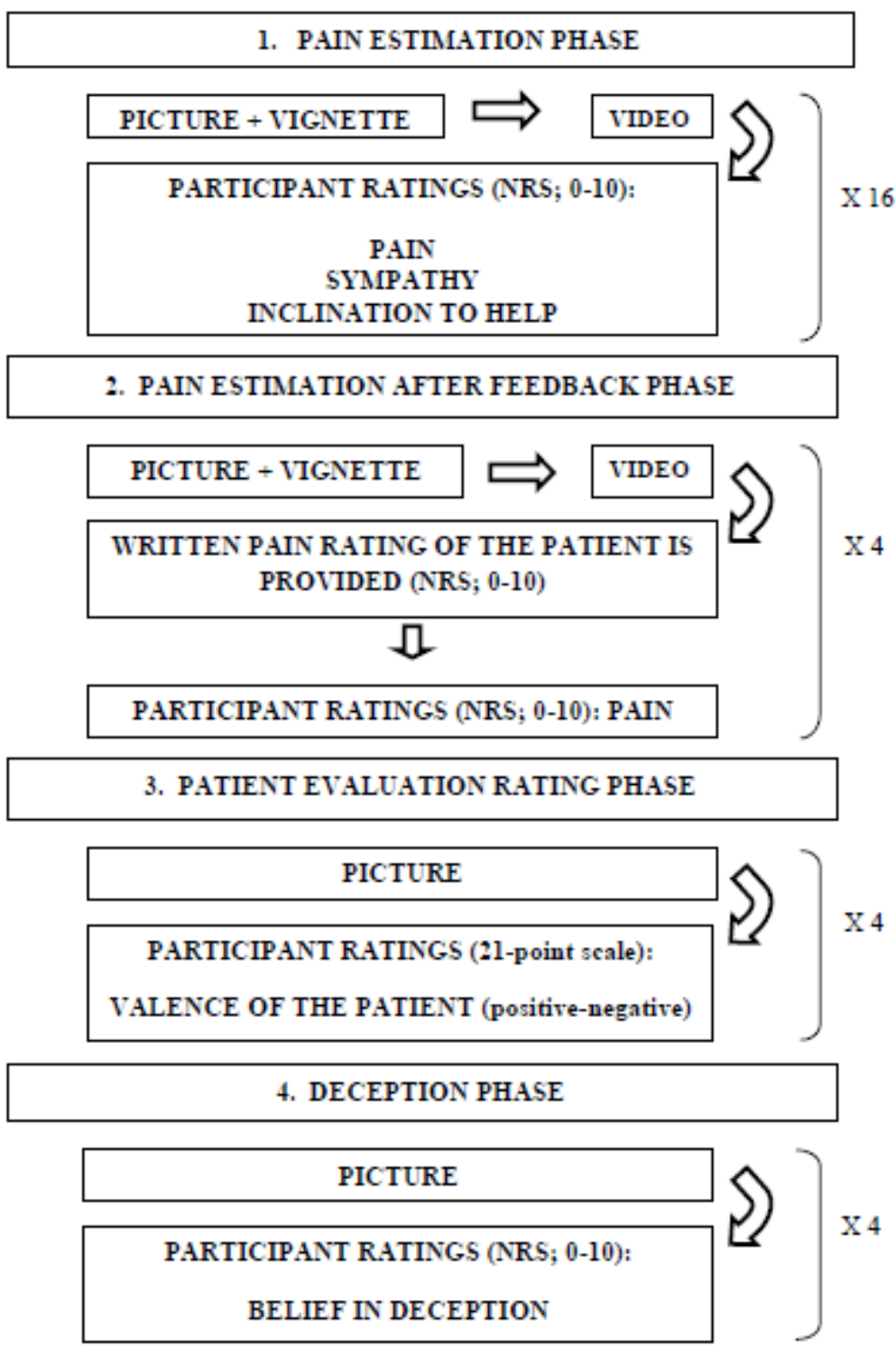


Figure 2. The three way interaction effect between patient's pain expression, medical evidence and psychosocial influences upon participant's inclination to help the patient with daily activities.

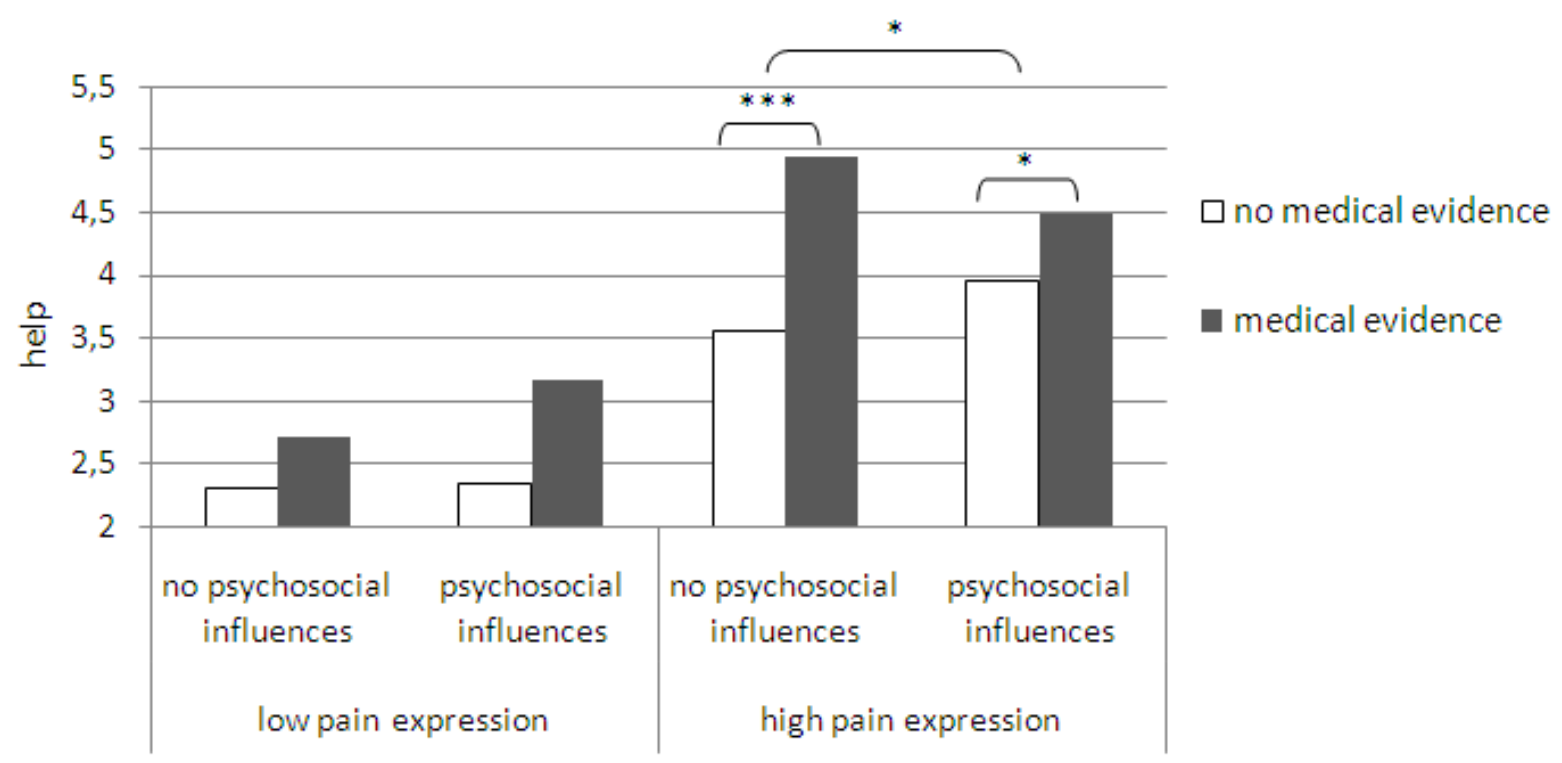

